\title{
SINGLE TEMPERATURE SCAN DETERMINATION OF DEFECT PARAMETERS IN DLTS EXPERIMENT*
}

\author{
L. DobACZEWSKI ${ }^{a}, Z$. KANCLERIS ${ }^{b}$, K. Bonde NIELSEN ${ }^{c}$ \\ AND A.R. PEAKER ${ }^{d}$ \\ anstitute of Physics, Polish Academy of Sciences \\ Al. Lotników 32/46, 02-668 Warsaw, Poland \\ ${ }^{b}$ Semiconductor Physics Institute, Vilnius, Lithuania \\ ${ }^{c}$ Institute of Physics and Astronomy, University of Aarhus, Aarhus, Denmark \\ ${ }^{d}$ Centre for Electronic Materials, University of Manchester \\ Institute of Science and Technology, Manchester, UK
}

Many point and extended defects in silicon, and other semiconducting materials, have been relatively well characterised by the standard DLTS technique. In this method the activation energy of carrier emission from the defect is calculated after multiple temperature scans. In this paper we demonstrate a new approach to the technique, in which after a single temperature scan the complete Arrhenius plot can be constructed for defects present in the sample with considerable concentrations. This method is much faster, accurate, and offers a much higher resolution in comparison with the standard DLTS method.

PACS numbers: $68.55 . \mathrm{Ln}, 71.55 . \mathrm{Cn}, 73.40 . \mathrm{Lq}$

In last few years there has been a number of attempts to improve the resolution of deep level transient spectroscopy (DLTS). In achieving this the standard boxcar or lock-in techniques have been abandoned and more emphasis has been put on the numerical analysis of the capacitance transients. In most cases this is done during sample temperature ramping, and simple numerical methods used to demonstrate the transient time constants (see, e.g. Ref. [1], and references therein). In general, these approaches neglect the homogeneous broadening of the defect parameters caused by varying the temperature. More elaborated numerical methods can be only used effectively when the sample is kept at constant temperature [2]. In the work reported here we present the results of a fast numerical method which is able to decompose the capacitance transient into a number of discrete components. The results of this procedure allow to construct the complete Arrhenius plot for most of the defects in the sample in a single temperature scan.

The integration method used for the analysis of the transients is based on the algorithm proposed by Tittelbach-Helmrich [3]. Basically, the number of exponents

*This work is supported in part by the European Community grant No. CIPA-CT94-0172. 
$(M)$ (discrete components) is not known beforehand, thus the calculations are performed by increasing $M$ beginning with $M=0$. As a criterion for finding the "right" solution a few empirically determined rules are used: (i) an increase in the number of exponents that are extracted from the transient must improve the fit between the calculated and initial signal; (ii) any calculated emission rate has to be positive; (iii) any amplitude of the component must be bigger than the noise. If the new solution calculated for $M+1$ exponent meets all the above-mentioned requirements it is accepted, otherwise it is rejected and parameters calculated one step before are accepted as the final result of transient analysis.

Numerical tests showed that the efficiency of the algorithm is different for a different number of components and noise-to-signal ratios (NSR). If the transient has only one exponential component this component is properly revealed even from very noisy signals (NSR can approach 0.3 ), however, to get reliable parameters for three exponential components one needs NSR to be smaller than $10^{-3}$. Most of the defect studied for testing the system had relatively high concentrations thus the criterion for good NSR has been fulfilled for the case of reliable extraction of at least two exponential components in the transients. When the method is applied during the measurements all results which do not follow the NSR threshold rule obtained from the tests are not accepted and not included in the spectrum. Numerical tests also showed that although the criterion for NSR seems to be rather rigid, the improvement in the resolution offered by our method in comparison with the standard DLTS is substantial. For satisfactory NSR the Tittelbach-Helmrich algorithm can, for example, distinguish two equal amplitudes when the difference in the emission rates is only $25 \%$.

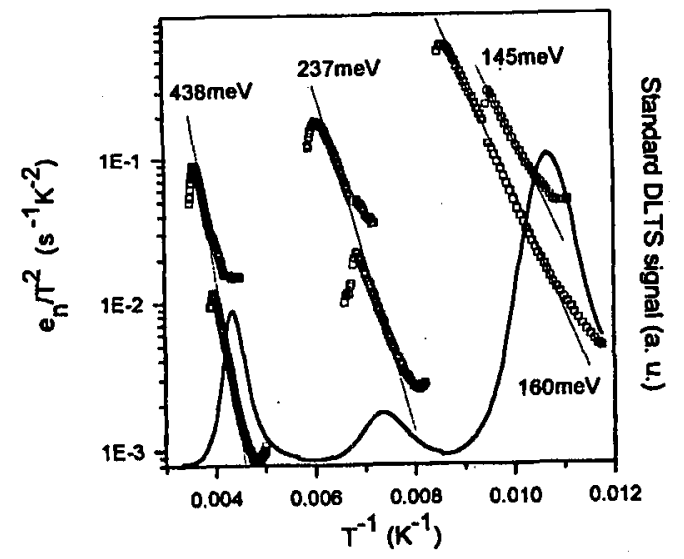

Fig. 1. The Arrhenius plot of the results of the single temperature scan for a sample of the proton irradiated $n$-type silicon. Squares are the results of the Tittelbach-Helmrich numerical algorithm (note that the points, which do not follow the noise-to-signal ratio acceptance criterion mentioned in the text, were removed from the figure). Straight lines are fits giving activation energies of the thermal emission processes. The solid line is the standard DLTS spectrum calculated during the same experiment. 
Figure 1 shows the results of the standard DLTS experiment compared with the results of the Tittelbach-Helmrich algorithm applied for the same set of the capacitance transients. The sample is an epitaxial layer of silicon doped with phosphorus, proton irradiated and annealed. In order to be able to overlay the conventional DLTS spectrum from the sample with the Arrhenius plot this spectrum is shown in the reciprocal temperature scale. The defect population is typical of a sample having this type of the technological history. At low temperature (right-hand side of the conventional spectrum) there is a peak assigned as the A-centre (oxygen-vacancy complex), at higher temperatures there is a peak related to the E-centre (phosphorus-vacancy complex). Between these two there is another peak with a much lower concentration, probably related to the phosphorus-carbon pair.

The Tittelbach-Helmrich algorithm revealed two exponential components present in the transients throughout the almost whole temperature range $(80-300 \mathrm{~K})$. In most of the temperatures there was one component with a time constant which did not change with the temperature and had rather low amplitude. We attribute this signal to the electronic response of the measuring system. The time constant of the other component varied with temperature showing in the temperature ranges, where there are standard DLTS peaks, very distinct activation behaviour. The activation energy of the signal around the E-centre peak was found to be $438 \mathrm{meV}$. Despite the more elaborated studies of this signal performed with the use of the Laplace transform DLTS [2] showing that this signal in reality consists of two components [4] (phosphorus-vacancy and vacancy-vacancy complexes) our studies of the same did not indicate this to be the case. However, the signal related to the A-centre having no apparent structure in the standard DLTS spectrum showed two distinct components when using the new method slightly differing in the activation energies (160 and $145 \mathrm{meV}$ ) and differing in amplitudes (ratio 5:1, respectively). The stronger signal can be related to the oxygen-vacancy complex while the shallower signal is probably related to the $(+/++)$ charge state of the thermal donor.

After examining different type of samples and comparing the single temperature scan with standard DLTS spectra we conclude that with our method new features in the spectra can be revealed. We observed a number of cases where in the standard DLTS spectra defects with a similar emission characteristics overlap which may lead to an incorrect defect assignment. All qualitative conclusions obtained by this new method are independently confirmed by the use of a more elaborated and well-established high resolution Laplace transform deep level spectroscopy.

\section{References}

[1] W.A. Doolittle, A. Rohatgi, J. Appl. Phys. 75, 4560 (1994).

[2] L. Dobaczewski, P. Kaczor, I.D. Hawkins, A.R. Peaker, J. Appl. Phys. 76, 194 (1994).

[3] K. Tittelbach-Helmrich, Meas. Sci. Technol. 4, 1323 (1993).

[4] K. Bonde Nielsen, L. Dobaczewski, unpublished. 